\title{
AIAA 2000-3902
}

\section{Low Order Equivalent System Identification for the Tu-144LL Supersonic Transport Aircraft}

Eugene A. Morelli

NASA Langley Research Center Hampton, VA

\section{AIAA Atmospheric Flight Mechanics Conference} August 14-17, 2000 / Denver, Colorado 


\title{
LOW ORDER EQUIVALENT SYSTEM IDENTIFICATION FOR THE Tu-144LL SUPERSONIC TRANSPORT AIRCRAFT
}

\author{
Eugene A. Morelli* \\ NASA Langley Research Center \\ Hampton, Virginia USA 23681-2199
}

\begin{abstract}
Low order equivalent system models were identified from flight test data for the Tu-144LL supersonic transport aircraft. Flight test maneuvers were executed by Russian and American test pilots flying the aircraft from Zhukovsky airfield outside Moscow, Russia. Flight tests included longitudinal and lateral / directional maneuvers at supersonic cruise flight conditions. Piloted frequency sweeps and multi-step maneuvers were used to generate data for closed loop low order equivalent system modeling. Model parameters were estimated using a flexible. high accuracy Fourier transform and an equation error / output error $(\mathrm{EE} / \mathrm{OE})$ formulation in the frequency domain. Results were compared to parameter estimates obtained using spectral estimation and subsequent least squares fit to frequency response data in Bode plots. Modeling results from the two methods agreed well for both a frequency sweep and multiple concatenated multi-step maneuvers. For a single multi-step maneuver, the EE/OE method gave a better model fit with improved prediction capability. A summary of closed loop low order equivalent system identification results for the Tu-144LL, including estimated parameters, standard errors, and flying qualities level predictions, were computed and tabulated.

$\begin{array}{ll} & \text { Nomenclature } \\ e & \text { equation error } \\ \text { EXT } & \text { extended } \\ \text { FQ } & \text { Flying Qualities }\end{array}$

* Rescarch Engineer. Dynamics \& Control Branch, Senior Member

Copyright 92000 by the American Institute of Aeronautics and Astronautics, Inc. No copyright is asserted in the United States under Title 17. U.S. Code. The U.S. Government has a royaltyfree license to exercise all rights under the copyright claimed herein for Governmental purposes. All other rights are reserved by the copyright owner.
\end{abstract}

\begin{tabular}{|c|c|}
\hline$h$ & altitude. $\mathrm{ft}$ \\
\hline HSR & High Speed Research \\
\hline$I_{x}, I_{y}, I_{z}$ & body-axis moments of inertia, slug- $\mathrm{ft}^{2}$ \\
\hline$j$ & imaginary number $\sqrt{-1}$ \\
\hline$J$ & cost function \\
\hline LOES & Low Order Equivalent System \\
\hline$M$ & Mach number \\
\hline$m$ & number of discrete frequencies \\
\hline$p, q, r$ & roll, pitch, and yaw rates, deg/sec \\
\hline RET & retracted \\
\hline$s$ & Laplace transform variable \\
\hline$t$ & time, sec \\
\hline$T_{R}$ & roll mode time constant, sec \\
\hline$u$ & input \\
\hline v & output error \\
\hline$w$ & weight. lbf \\
\hline$x_{i g}$ & longitudinal center of gravity position \\
\hline$y$ & model output \\
\hline$z$ & measured output \\
\hline$\alpha$ & angle of attack, deg \\
\hline$\eta$ & pilot control deflection, $\mathrm{cm}$ \\
\hline$\tau$ & equivalent time delay, sec \\
\hline$\omega$ & frequency, $\mathrm{rad} / \mathrm{sec}$ \\
\hline$\zeta$ & damping ratio \\
\hline$\angle$ & phase angle, deg \\
\hline & magnitude of a complex number \\
\hline
\end{tabular}

\section{$\underline{\text { superscripts }}$}

$\doteqdot \quad$ complex conjugate transpose

estimate

- Laplace or Fourier transform

$-1 \quad$ matrix inverse 
subscripts

$\begin{array}{ll}a & \text { lateral stick } \\ D R & \text { Dutch Roll } \\ e & \text { longitudinal stick } \\ E E & \text { Equation Error } \\ L O E S & \text { Low Order Equivalent System } \\ L S & \text { Least Squares } \\ O E & \text { Output Error } \\ o & \text { nominal or trim value } \\ r & \text { rudder pedal } \\ S P & \text { Short Period }\end{array}$

\section{Introduction}

As part of the NASA High Speed Research (HSR) program, the Tupolev Aircraft Company in Russia was commissioned to refurbish, re-engine, and instrument an out-of-service Tu-144D supersonic transport aircraft, for use as a supersonic flying laboratory. The modified aircraft, designated the Tu-144LL, was operated from Zhukovsky airfield, near Moscow, Russia.

Flight test data was collected for 6 experimental investigations related to high speed research. One of the experimental investigations was closed loop flying qualities. Flight testing began with 19 Phase I flights in 1996-1997, flown exclusively by Tupolev pilots.

Ref. [1] contains results of longitudinal, lateral, and directional closed loop Low $\underline{\text { Order Equivalent }}$ System (LOES) modeling based on the Phase I flight test data, using an equation error method in the frequency domain ${ }^{1-3}$. The LOES model characterizes the linearized dynamic closed loop response of the airframe and control system as it appears to the pilot. The form of the LOES model is the same as that for an open loop unaugmented airplane with classical dynamic modes, except the inputs are pilot controls with equivalent time delays, instead of control surface deflections. Equivalent time delay is introduced to account for time delay resulting from the control system implementation (e.g., sampling delay), and the phase lag at high frequency from control system dynamics and various nonlinearities, such as control surface rate limiting.

In the past, the majority of flight testing for flying qualities evaluation and specification compliance was done using unaugmented aircraft with classical dynamic modal responses. To apply specifications developed for unaugmented airplanes to airplanes whose dynamics are no longer classical because of additional dynamics from the control system, the concept of a Low Order Equivalent System (LOES) model was introduced ${ }^{4-6}$.

If the closed loop response of an augmented airplane to pilot inputs can be accurately characterized by a LOES model, then specifications for classical unaugmented aircraft parameters found in the current Military Specification for Flying Qualities of Piloted Airplanes $^{4}$ (hereafter called the Mil-Spec) can be applied directly to the estimated parameters in the LOES model. The Mil-Spec quantifies the relationship between parameter values in a low order dynamic model and pilot opinion, as measured by flying qualities levels ${ }^{4}$, which are based on Cooper-Harper ratings ${ }^{5}$. The Mil-Spec also contains specifications relating flying qualities levels and equivalent time delay. LOES models for the aircraft closed loop dynamic response are fit over the frequency band corresponding to typical pilot inputs, $0.1-10 \mathrm{rad} / \mathrm{sec}$.

Many flight test research programs ${ }^{4.6-9}$ have demonstrated that the LOES concept can be used to predict pilot opinion (quantified by flying qualities level) for augmented aircraft dynamic response that is in reality high order and/or nonlinear. LOES models identified from flight test data are also useful for validating linear control law designs and verifying specification compliance, since the LOES model represents the achieved linearized closed loop dynamics of the aircraft. LOES models can also be used for rudimentary simulation within the limited flight envelope where the model is valid.

A second phase of flight testing for closed loop flying qualities research was conducted on the Tu-144LL in the fall of 1998. Phase II testing included four flights - one subsonic flight with an all Russian crew (flight 20), one subsonic flight with an American evaluation pilot (flight 21), and two supersonic flights with American evaluation pilots (flights 22 and 23). These flights included flying qualities evaluation tasks. approaches, and special maneuvers designed to collect data for closed loop LOES modeling. Ref. [10] contains extensive information on the Tu-144LL aircraft, flight profiles, and maneuvers for these flights, including qualitative pilot commentary and narrative flight summaries written by the American evaluation pilots.

This work examined LOES modeling based on the Tu-144LL Phase II flight test data using an equation error / output error technique in the frequency domain with a flexible, high accuracy Fourier transform ${ }^{2}$. Results were compared with LOES models 
obtained from a least squares fit to an empirical estimate of the Bode plot, using commercially available software called CIFER ${ }^{\circledR 11-13}$. Correlation of the analytical LOES modeling results with pilot opinion was not done, because the pilots gave only qualitative narrative assessments, and not Cooper-Harper ratings.

The next section describes the two LOES model parameter estimation techniques used. Next, LOES modeling issues are examined by applying the two parameter estimation techniques to Tu-144LL Phase II flight test data. Finally, LOES modeling results for the Tu-144LL Phase II flights are computed and tabulated.

\section{Methods}

The model structure for LOES modeling is fixed a priori to correspond to classical linear aircraft dynamics with an input time delay. For the short period longitudinal dynamic mode, the closed loop pitch rate response to longitudinal stick deflection is modeled in transfer function form as ${ }^{4}$

$$
\tilde{q}=\frac{K_{\theta}\left(s+1 / T_{\theta_{2}}\right) e^{-\tau_{r^{\prime}} s}}{\left(s^{2}+2 \zeta_{S P} \omega_{S P} s+\omega_{S P}^{2}\right)} \tilde{\eta}_{\ell}
$$

The current Mil-Spec ${ }^{4}$ correlates pilot opinion, quantified by flying qualities levels from the Cooper-Harper scale ${ }^{5}$, with ranges of values for all model parameters in Eq. (1), except $K_{\dot{\theta}}$.

For lateral / directional closed loop dynamics, the LOES model for roll rate response is

$$
\begin{aligned}
\tilde{p}= & \frac{K_{p_{1}}\left(s^{2}+2 \omega_{p_{1}} \zeta_{m_{1}} s+\omega_{p_{1}}^{2}\right) e^{-\tau_{r} s}}{\left(s+1 / T_{R}\right)\left(s^{2}+2 \zeta_{D R} \omega_{D R} s+\omega_{D R}^{2}\right)} \tilde{\eta}_{r} \\
& +\frac{K_{p_{2}}\left(s^{2}+2 \omega_{m_{2}} \zeta_{p_{2}} s+\omega_{m_{2}}^{2}\right) e^{-\tau_{a} s}}{\left(s+1 / T_{R}\right)\left(s^{2}+2 \zeta_{D R} \omega_{D R} s+\omega_{D R}^{2}\right)} \tilde{\eta}_{a}
\end{aligned}
$$

For closed loop yaw rate response. the LOES model is

$$
\begin{aligned}
\tilde{r}= & \frac{K_{r_{1}}\left(s^{2}+2 \omega_{r_{1}} \zeta_{r_{1}} s+\omega_{\eta_{1}}^{2}\right) e^{-\tau_{r} s}}{\left(s+1 / T_{R}\right)\left(s^{2}+2 \zeta_{D R} \omega_{D R} s+\omega_{D R}^{2}\right)} \tilde{\eta}_{r} \\
& +\frac{K_{r_{2}}\left(s^{2}+2 \omega_{r_{2}} \zeta_{r_{2}} s+\omega_{r_{2}}^{2}\right) e^{-\tau_{a} s}}{\left(s+1 / T_{R}\right)\left(s^{2}+2 \zeta_{D R} \omega_{D R} s+\omega_{D R}^{2}\right)} \tilde{\eta}_{a}
\end{aligned}
$$

The forms of the models in Eqs. (2) and (3) follow from the closed loop lateral / directional state space model, omitting the gravity term in the side force equation.

The problem addressed in this work is accurate estimation of the model parameters in Eqs. (1)-(3) using measured input-output tlight test data. The idea is to match the measured outputs or output time derivatives with the corresponding quantities from the model by adjusting model parameters to minimize a measure of fit error, which is the sum of the squared deviations between model quantity and measured quantity. Several methods exist for estimating model parameters based on measured data, both in the time domain $^{14,15}$ and the frequency domain ${ }^{1-4.11-13}$.

For identification purposes. Eqs. (1)-(3) can be re-parameterized as

$$
\begin{gathered}
\tilde{q}=\frac{\left(b_{1} s+b_{0}\right) e^{-\tau_{t^{\prime}} s}}{\left(s^{2}+a_{1} s+a_{0}\right)} \tilde{\eta}_{e} \\
\tilde{p}=\frac{\left(d_{2} s^{2}+d_{1} s+d_{0}\right) e^{-\tau_{a} s}}{\left(s^{3}+c_{2} s^{2}+c_{1} s+c_{0}\right)} \tilde{\eta}_{a} \\
+\frac{\left(e_{2} s^{2}+e_{1} s+e_{0}\right) e^{-\tau_{r} s}}{\left(s^{3}+c_{2} s^{2}+c_{1} s+c_{0}\right)} \tilde{\eta}_{r} \\
\tilde{r}=\frac{\left(f_{2} s^{2}+f_{1} s+f_{0}\right) e^{-\tau_{r} s}}{\left(s^{3}+c_{2} s^{2}+c_{1} s+c_{0}\right)} \tilde{\eta}_{r} \\
+\frac{\left(g_{2} s^{2}+g_{1} s+g_{0}\right) e^{-\tau_{d} s}}{\left(s^{3}+c_{2} s^{2}+c_{1} s+c_{0}\right)} \tilde{\eta}_{a}
\end{gathered}
$$

The relationship among the parameters in Eqs. (1)-(3) and Eqs. (4)-(6) can be found by direct comparison. All LOES modeling was done in the frequency domain, because the equivalent time delay parameters can be estimated more easily and accurately in the frequency domain 2.11 .

Two methods were used to estimate the parameters in Eqs. (4)-(6) based on flight test data. The first method, described in Refs. [11]-[13], involves computing a non-parametric estimate of the frequency response in the form of a Bode plot. Then, the parameters in the models of Eqs. (4)-(6) are adjusted to minimize the least squares cost function: 


$$
\begin{aligned}
J_{L S}= & \sum_{i=1}^{m}\left(20 \log _{10}\left|\frac{\tilde{z}\left(\omega_{i}\right)}{\tilde{u}\left(\omega_{i}\right)}\right|-20 \log _{10}\left|\frac{\tilde{y}\left(\omega_{i}\right)}{\tilde{u}\left(\omega_{i}\right)}{ }_{\text {LOES }}\right|\right)^{2} \\
& +0.0175\left(\angle\left\{\frac{\tilde{z}\left(\omega_{i}\right)}{\tilde{u}\left(\omega_{i}\right)}\right\}-\angle\left\{\frac{\tilde{y}\left(\omega_{i}\right)}{\tilde{u}\left(\omega_{i}\right)}{ }_{\text {LOES }}\right\}\right)^{2}
\end{aligned}
$$

Typically, the number of frequencies $m$ is 50 , and the frequencies are chosen logarithmically on the interval $[0.1,10] \mathrm{rad} / \mathrm{sec}$. Commercially available FORTRAN software called CIFER ${ }^{\circledR 11-1.3}$ was used to estimate LOES model parameters with this method.

The second method, described in Ref. [2], is a two-step approach using equation error (EE) and output error (OE) formulations in the frequency domain for the parameter estimation. For the equation error formulation, the cost function is:

$$
\begin{aligned}
J_{E E} & =\frac{1}{2} \sum_{i=1}^{m}\left|D\left(j \omega_{i}\right) \tilde{z}_{i}-N\left(j \omega_{i}\right) \tilde{u}_{i} e^{-j \omega_{i} \tau}\right|^{2} \\
& =\frac{1}{2} \sum_{i=1}^{m}\left|\tilde{e}_{i}\right|^{2}=\frac{1}{2}\left(\tilde{\boldsymbol{e}}^{\dagger} \tilde{\boldsymbol{e}}\right)
\end{aligned}
$$

where $N\left(j \omega_{i}\right)$ and $D\left(j \omega_{i}\right)$ are the numerator and denominator polynomials of the transfer function, with $s=j \omega_{i}$. Accurate Fourier transforms for $m$ arbitrary frequencies were computed using the method of Ref. [16]. Parameter estimation results from the equation error method were used as starting values for the output error method, which minimized the cost function:

$$
J_{O E}=\frac{1}{2} \sum_{i=1}^{m}\left|\tilde{z}_{i}-\tilde{y}_{i}\right|^{2}=\frac{1}{2} \sum_{i=1}^{m}\left|\tilde{v}_{i}\right|^{2}=\frac{1}{2}\left(\tilde{v}^{\dagger} \tilde{v}\right)
$$

Final parameter estimates were from the output error formulation, because of known favorable asymptotic properties ${ }^{14,15}$. Equation error is much more robust to starting values of the parameters, because the model output depends linearly on all model parameters except the equivalent time delay $\tau$. In this work, the equation error method always converged quickly and without difficulty using zeros for the starting values of all the parameters. Parameter estimates from the equation error method provide good starting values for the output error method. The output error method needs good starting values for the parameters because the model output depends nonlinearly on the transfer function denominator parameters, in addition to the equivalent time delay $\tau$.
The EE/OE sequence therefore gives parameter estimation results with good asymptotic properties, without requiring good starting values for the parameters. Estimated parameter standard errors do not require correction, because the analysis in the frequency domain automatically accounts for colored residuals $2,14.15$.

Both the equation error and output error methods are nonlinear estimation problems, because of the equivalent time delay parameter. Details on the optimization methods used to find both equation error and output error parameter estimates can be found in Ref. [2].

Any of the methods described above can be used for general multi-input, multi-output models. For the results given here, Eqs. (4)-(6) were used to identify LOES models from flight test data.

Important differences in the two methods can be appreciated by examining the cost functions in Eqs. (7)-(9). The CIFER ${ }^{\circledR}$ method minimizes a cost comprised of two parts - the squared difference between measured and model transfer function frequency response magnitude in $\mathrm{dB}$ and phase in deg. The first step in the CIFER ${ }^{\circledR}$ method is non-parametric estimation of the transfer function frequency response in the form of a Bode plot, obtained using the ratio of estimates for the input-output cross-spectral density and input auto-spectral density. The two weighted terms in the cost involve nonlinear functions of the complex frequency response (gain in $\mathrm{dB}$ and phase angle in deg). Since the frequency response estimate is calculated from a ratio of spectral estimates, the accuracy of the estimated frequency response can degrade when the frequency content of the data is sparse. CIFER ${ }^{\circledR}$ uses coherence weighting in the parameter estimation to emphasize frequency domain data with good linearity and signal-to-noise ratio. The EE/OE method uses high accuracy, arbitrary frequency Fourier transforms ${ }^{16}$ directly, and minimizes the square magnitude of the equation error (EE) or the output error $(\mathrm{OE})$, with equal weighting of the real and imaginary parts of the complex quantities in the cost. Spectral components in the data, whether sparse or not, influence parameter estimation results according to their complex magnitude in the frequency domain.

Details of the calculations and optimization methods used to find the parameter estimates that minimized the cost functions in Eqs. (7)-(9) can be found in Refs. [11]-[13] for the CIFER ${ }^{\circledR}$ method, and Ref. [2] for the EE/OE method. 


\section{Aircraft}

The Tu-144LL test aircraft is a modified version of the Tupolev Tu- 144D cranked delta wing supersonic transport. Modifications included installation of Kuznetsov NK-321 engines rated at 31,000 lbf static dry thrust and 55,000 lbf in afterburner at sea level, and installation of flight-test quality instrumentation with sampling rate $32 \mathrm{~Hz}$. A 3-view drawing of the Tu-144LL appears as Figure 1. Vehicle characteristics are summarized in Table 1. For cruise flight, the canards are retracted along the fuselage above and behind the cockpit, and the nose is raised. On approach, the canards are extended for slow flight and the nose is drooped for enhanced visibility from the cockpit. Flight crew consisted of two pilots, a navigator, and a flight engineer. Details of the flight control system are available in Ref. [10].

Air data on the Tu-144LL was obtained from production sensors, not specialized flight test instrumentation, such as an air data probe. Data compatibility analysis 17 indicated that measurements of angle of attack and sideslip angle contained large systematic errors, including lag and hysteresis effects. Consequently, the flight test data analysis was done using only body-axis angular rate data for the measured outputs. The angular rate data was found to be consistent with measured Euler angle data, using data compatibility analysis with the rotational kinematic equations only ${ }^{17}$. Finally, there was some evidence to suggest that time skews were present in the measured input-output data, due to the instrumentation system. The main clue was an excessive time delay $(\sim 200 \mathrm{msec})$ between initial movement of control surfaces and angular rate response. However, it could not be determined conclusively what, if anything, the time skews were, so the data was analyzed as recorded.

\section{$\underline{\text { Results }}$}

The first case studied was closed loop identification of the Tu-144LL longitudinal short period LOES dynamics for supersonic cruise flight at Mach 1.93. The pilot applied a frequency sweep input to the longitudinal stick, shown in the upper plot of Figure 2. Measured pitch rate response is shown as the solid line in the middle plot of Figure 2. The dotted line on the same plot shows the computed pitch rate response to measured stick input, using the LOES model of Eq. (4) with parameters estimated using the $\mathrm{EE} / \mathrm{OE}$ method. The lower plot in Figure 2 shows the output residual, which is the difference between the measured and computed pitch rate traces shown in the middle plot of Figure 2. Frequencies used in the data analysis were evenly spaced at $0.1 \mathrm{rad} / \mathrm{sec}$ on the interval $[0.1,10] \mathrm{rad} / \mathrm{sec}$. The model fit in the frequency domain (not shown) was excellent, and Figure 2 shows that the model fit in the time domain was also excellent. Estimated parameter values and standard errors for the identified $\tilde{q} / \tilde{\eta}_{e}$ LOES model are listed in column 2 of Table 2. Estimated standard errors in Table 2 indicate that the model parameter accuracy was better than $8 \%$, based on parameter estimate values, for all $\tilde{q} / \tilde{\eta}_{e}$ LOES model parameters.

CIFER $^{\circledR}$ was used with the same data to estimate model parameters in the same LOES model of Eq. (4). Results are given in the third column of Table 2. Comparison of the values in columns 2 and 3 of Table 2 indicates that the results from CIFER $^{\circledR}$ agreed well with corresponding values from the EE/OE method. This built confidence in the results, because the two methods differ in formulation, cost function. and optimization technique, but gave similar LOES modeling results. Since the CIFER ${ }^{\circledR}$ model parameters were so close to the EE/OE model parameters, the compuled pitch rate response from the CIFER $\left.{ }^{(}\right)$model was nearly the same as the computed pitch rate response from the EE/OE model, shown by the dotled line in Figure 2.

The upper plot of Figure 3 shows a different kind of longitudinal stick input implemented by the pilot at approximately the same flight condition. This input was a modified doublet called a 2-1-1, which ideally consists of alternating pulses with pulse widths in the ratio 2-1-1. Inputs of this type are also called multi-step inputs. The widths of the pulses were chosen so that the frequencies corresponding to the "2" and " 1 " pulses bracketed the a priori estimate of the natural frequency for the closed loop short period mode. Data analysis results from Phase I tlights ${ }^{1}$ at transonic cruise conditions were used to design the input pulse width and amplitude.

The EE/OE and CIFER ${ }^{\circledR}$ methods were used to estimate LOES model parameters in Eq. (4), hased on data from the 2-1-1 maneuver. Table 3 contains the results. The EE/OE method produced LOES model parameter estimates close to those estimated using the frequency sweep input at approximately the same flight condition (cf. the second column of Tables 2 and 3 ). Some discrepancies in the parameter estimates may be due to slight differences in flight condition and mass properties. The lower plot in Figure 3 shows a good time domain match to the measured pitch rate for the EE/OE model. CIFER ${ }^{(\mathbb{B}}$ parameter estimates in column 3 of Table 3 were different than the EE/CE values, 
mainly because the 2-1-1 data did not provide sufficient power across the LOES modeling frequency range to allow an accurate Bode plot estimate from spectral estimates. Figure 3 shows the corresponding time domain mismatch to the pitch rate measurement for the CIFER ${ }^{\circledR}$ model based on data from 2-1-1 maneuver 22_4f only, denoted by CIFER $4 f$.

The CIFER ${ }^{\circledR}$ method was not designed to work with data of this type. However, results from CIFER ${ }^{\circledR}$ improved and approached the EE/OE results when additional 2-1-1 maneuvers at the same flight condition were concatenated for the analysis, allowing sufficient averaging from the data windows for accurate spectral estimation. Additional 2-1-1 maneuvers 22_4a and 22 4b were concatenated to the original single maneuver 22_4f for another CIFER ${ }^{\circledR}$ analysis. The results shown in column 4 of Table 3 are in good agreement with the EE/OE results, and Figure 3 shows a good match to the pitch rate measurement. The multiple-maneuver CIFER $^{\circledR}$ results are denoted by CIFER 4abf. The CIFER ${ }^{\circledR}$ method therefore works well for 2-1-1 maneuvers also, given sufficient data for good spectral estimates. The EE/OE method is less sensitive to the spectral content in the data, because Fourier transforms are used directly, instead of spectral estimates. This allowed the EE/OE method to accurately identify LOES models using data from either a frequency sweep or a single 2-1-1 maneuver.

The EE/OE method can also be used with multiple maneuvers. For this case, results from the $\mathrm{EE} / \mathrm{OE}$ method using concatenated data from maneuvers 22_4a, 22_4b, and 22_4f were similar to those given in column 2 of Table 3 , which were based on data from maneuver 22_4f only.

Flight time required for the 2-1-1 maneuver is approximately $1 / 6$ the time required for the frequency sweep maneuver. It might be possible to shorten the frequency sweep maneuver and maintain its effectiveness by omitting the lower frequency portions of the maneuver, since only the relatively high frequency closed loop short period dynamics were being identified.

Figure 4 shows a similar 2-1-1 maneuver executed at approximately the same flight condition. In the lower plot of Figure 4, the LOES models identified in the previous example (cf. Table 3) were used with the input shown in the top of Figure 4 to predict the pitch rate response. For this prediction case, the fidelity of the EE/OE and CIFER ${ }^{\circledR}$ models was similar to what was seen in Figure 3.
Figure 5 shows another longitudinal 2-1-1 maneuver, this time at transonic cruise, Mach 0.88 . The model trace shown in the lower plot of Figure 5 was generated using the LOES model of Eq. (4) with parameters estimated using the EE/OE method. Results for this case are given in column 3 of Table 4. In Figure 6, the identified LOES model was used for a prediction case at the same flight condition, with inverted polarity of the 2-1-1 longitudinal stick input. Figure 6 demonstrates the excellent prediction capability for LOES models identified using the EE/OE method, and also indicates that the LOES model was not sensitive to input polarity for this flight condition.

The final example was multiple-input, multipleoutput lateral / directional LOES modeling at supersonic cruise. Roll rate and yaw rate outputs were used with both lateral stick and rudder pedal inputs, in the model structure of Eqs. (5) and (6). Data from both a rudder pedal sweep and a lateral stick sweep maneuver were combined in the frequency domain for LOES modeling using the EE/OE method. The inputoutput data in Figure 7 is from the lateral stick sweep maneuver. The model fit to the data in the time domain was excellent, demonstrating that the EE/OE method can be successfully used for multiple-input, multiple-output lateral / directional LOES modeling. The quality of the time domain fit to the rudder pedal sweep maneuver (not shown) was similar. The last column of Table 4 contains results for this case.

Longitudinal and lateral / directional LOES modeling results for Tu-144LL Phase II flights are summarized in Table 4. Flight conditions included are: canard-extended approach at Mach 0.34, transonic cruise at Mach 0.88, and supersonic cruise at Mach 1.93. All LOES modeling results in Table 4 were obtained using the EE/OE method. More than one maneuver was used in cases where the flight conditions for the maneuvers were nearly the same. This is indicated by the maneuver(s) listed for each flight condition. Flying qualities level predictions using the Mil-Spec were level 2 or 3 because of large values for estimated equivalent time delay, $\mathrm{cf}$. Table 4 . Based on all LOES parameter estimates except the equivalent time delay, the Mil-Spec indicated that the aircraft had level 2 longitudinal flying qualities for supersonic cruise, transonic cruise, and canard-extended approach, and level 1 lateral / directional flying qualities for supersonic cruise. Flying qualities levels predicted exclusive of the equivalent time delay (i.e., level 2 longitudinal, and level 1 lateral / directional) were consistent with qualitative comments from the evaluation pilots ${ }^{10}$. This was further evidence of the possible instrumentation time skew mentioned earlier. 
Similar findings of excessive time delay were reported in Ref. [1] for Phase I flight test data analysis.

LOES modeling was also done using the $\mathrm{EE} / \mathrm{OE}$ method for several longitudinal cases with $150 \mathrm{msec}$ delay removed from the pitch rate measurement. This was the estimated time delay that could be reasonably attributed to instrumentation time skew based on control surface deflection and pitch rate measurements for the multi-step maneuvers, allowing for normal pitch rate response delay. For pitch rate data with $150 \mathrm{msec}$ time delay removed, estimated LOES model parameters were essentially unchanged, except that the equivalent time delay estimate was roughly $150 \mathrm{msec}$ smaller. If estimated equivalent time delays were reduced by $150 \mathrm{msec}$ for all longitudinal and lateral / directional cases in Table 4, the Mil-Spec indicated that the aircraft would have level 2 longitudinal flying qualities and level I lateral / directional flying qualities, again in concurrence with qualitative pilot opinion ${ }^{10}$.

\section{Concluding Remarks}

This work was concerned with accurately identifying closed loop Low Order Equivalent $\underline{\text { System }}$ (LOES) models from flight test data for the Tu-144LL supersonic transport aircraft. LOES models are linear models with input time delay, used to characterize closed loop aircraft response to pilot inputs. Data analysis was done in the frequency domain using two methods: a two-step equation error / output error technique (EE/OE) using a high accuracy Fourier transform with selectable frequency range and resolution, and a least squares fit to an empirical estimate of the Bode plot (CIFER $\left.{ }^{\circledR}\right)$. Both methods identified accurate LOES models with good prediction capability when used with data from a frequency sweep maneuver or multiple concatenated multi-step maneuvers. For a single multi-step maneuver, the EE/OE method gave a better model fit with improved prediction capability.

The EE/OE modeling technique was applied to Phase II flight test data from the Tu-144LL aircraft to identify closed loop LOES models at various flight conditions, including transonic cruise, canard-extended approach, and supersonic cruise. Longitudinal and lateral / directional LOES modeling results were computed and presented. Identified LOES models were consistent with qualitative pilot opinion. if equivalent time delays were adjusted for estimated instrumentation time skew.
The results shown here suggest that the EE/OE method can be used in conjunction with multi-step maneuvers to decrease flight test time required to collect data for LOES model identification. All LOES modeling was done using only measurements for pilot inputs and body-axis angular rate outputs, demonstrating that this type of analysis can be done successfully with minimal instrumentation.

\section{Acknowledgements}

Flight test maneuvers were flown by Sergei Borisov from Tupolev, Rob Rivers from NASA Langley, and Gordon Fullerton from NASA Dryden. CIFER ${ }^{\circledR}$ results for frequency sweep and multiple 2-1-1 maneuvers were provided by Dr. Mark Tischler from NASA Ames.

\section{References}

1. Curry, T.J.. "Estimation of Handling Qualities Parameters of the Tu-144 Supersonic Transport Aircraft from Flight Test Data", NASA CR-2000210290 . May 2000.

2. Morelli, E.A., "Identification of Low Order Equivalent System Models from Flight Test Data". NASA TM-2000-210117. May 2000.

3. Klein. V. "Aircraft Parameter Estimation in Frequency Domain". AIAA paper 78-1344. Atmospheric Flight Mechanics Conference. Palo Alto, CA. August 1978.

4. Military Standard - Flying Qualities of Piloted Aircraft. MIL-STD-1797A, January 1990.

5. Cooper, G.E., and Harper. R.P. Jr., "The Use of Pilot Rating in the Evaluation of Aircraft Handling Qualities". NASA TN D-5153. April 1969.

6. Hodgkinson, J.. LaManna. W.J.. and Heyde. J.L.. "Handling Qualities of Aircraft with Stability and Control Augmentation Systems - A Fundamental Approach", Aeronautical Journal, pp. 75-81. February 1976.

7. Mitchell, D.G. and Hoh. R.H., "Low-Order Approaches to High-Order Systems: Problems and Promises", Journal of Guidance, Control, and Dynamics, Vol. 5. No. 5, Sept.-Oct. 1982.

8. Neal, T.P.. and Smith, R.E., "An In-Flight Investigation to Develop Control System Design Criteria for Fighter Airplanes", AFFDL-TR-7074. Vols. I \& II, December 1970. 
9. Hodgkinson, J., Snyder, R.C., and Smith, R.E., "Equivalent System Verification and Evaluation of Augmentation Effects on Fighter Approach and Landing Flying Qualities, Vols. 1 \& 2", AFWALTR-81-3116, September 1981.

10. Rivers, R.A., Jackson, E.B., Fullerton, C.G., Cox, T.H., and Princen, N.H. "A Qualitative Piloted Evaluation of the Tupolev Tu-144 Supersonic Transport", NASA TM-2000-209850. February 2000.

11. Tischler, M.B.. Cauffman, M.G., "FrequencyResponse Method for Rotorcraft System Identification: Flight Applications to BO-105 Coupled Rotor/Fuselage Dynamics", Journal of the American Helicopter Society, Vol. 37, No. 3, pp. 3-17, July 1992.

12. Tischler, M.B. and Cauffman, M.G., "Comprehensive Identification from Frequency Responses, Vol. 1 - Class Notes", NASA CP 10149, September 1994.

13. Tischler, M.B. and Cauffman, M.G., "Comprehensive Identification from Frequency Responses, Vol. 2 - User's Manual", NASA CP 10150, September 1994.

14. Maine, R.E. and Iliff, K.W., "Application of Parameter Estimation to Aircraft Stability and Control - The Output-Error Approach", NASA RP 1168, June 1986.

15. Morelli, E.A. and Klein, V., "Determining the Accuracy of Maximum Likelihood Parameter Estimates with Colored Residuals", NASA CR 194893, March 1994.

16. Morelli, E.A. "High Accuracy Evaluation of the Finite Fourier Transform using Sampled Data", NASA TM 110340, June 1997.

17. Klein, V. and Morgan, D.R. "Estimation of Bias Errors in Measured Airplane Responses using Maximum Likelihood Method", NASA TM 89059, January 1987.

Table $1 \mathrm{Tu}-144 \mathrm{LL}$ Vehicle Characteristics

\begin{tabular}{cc}
\hline Length, $\mathrm{ft}$ & 215.5 \\
\hline Height, wheels up, $\mathrm{ft}$ & 42.2 \\
\hline Wing Span, $\mathrm{ft}$ & 94.5 \\
\hline Wing Area, $\mathrm{ft}^{2}$ & 6769 \\
\hline Mean Aerodynamic Chord, $\mathrm{ft}$ & 76.5 \\
\hline Max. Fuel, lbm & 209,440 \\
\hline Est. Max. Range, $\mathrm{nm}$ & 1,620 \\
\hline Max. Mach Number & 2.4 \\
\hline
\end{tabular}

Table 2 Tu-144LL Longitudinal Short Period LOES Modeling Results, Frequency Sweep Maneuver 23_1 a, $\alpha_{o}=4.8 \mathrm{deg}, M_{o}=1.93, x_{c g}=0.46 \bar{c}, I_{y}=1.44 \times 10^{7}$

\begin{tabular}{ccc}
\hline \hline & Maneuver 23_la \\
\hline Parameter & $\begin{array}{c}\text { EE/OE } \\
\text { Estimate } \\
\text { (Std. Error) }\end{array}$ & $\begin{array}{c}\text { CIFER }^{\circledR} \\
\text { Estimate } \\
\text { (Std. Error) }\end{array}$ \\
\hline$b_{1}$ & $\begin{array}{c}0.353 \\
(0.011)\end{array}$ & $\begin{array}{c}0.380 \\
(0.022)\end{array}$ \\
\hline$b_{0}$ & 0.106 & 0.107 \\
$(0.006)$ & $(0.013)$ \\
\hline$a_{1}$ & 0.932 & 1.040 \\
& $(0.035)$ & $(0.121)$ \\
\hline$a_{0}$ & 1.970 & 2.103 \\
$(0.035)$ & $(0.133)$ \\
\hline$\tau_{e}$ & 0.194 & 0.192 \\
\hline $1 / T_{\theta_{2}}$ & $0.014)$ & $(0.013)$ \\
\hline$\zeta_{S P}$ & 0.30 & 0.28 \\
\hline$\omega_{S P}$ & 1.40 & 0.36 \\
\hline \hline
\end{tabular}

Table 3 Tu-144LL Longitudinal Short Period LOES Modeling Results, 2-1-1 Maneuvers

\begin{tabular}{|c|c|c|c|}
\hline \multirow[b]{2}{*}{ Parameter } & \multicolumn{2}{|c|}{ Maneuver 22_4f } & \multirow{2}{*}{$\begin{array}{c}\begin{array}{c}\text { Maneuvers } \\
22 \_4 a, b, f\end{array} \\
\text { CIFER }^{\circledR} \\
\text { Estimate }\end{array}$} \\
\hline & $\begin{array}{c}\text { EE/OE } \\
\text { Estimate } \\
\text { (Std. Error) }\end{array}$ & $\begin{array}{l}\text { CIFER }^{\circledR} \\
\text { Estimate }\end{array}$ & \\
\hline$b_{1}$ & $\begin{array}{c}0.375 \\
(0.014) \\
\end{array}$ & 0.304 & 0.430 \\
\hline$b_{0}$ & $\begin{array}{c}0.114 \\
(0.008) \\
\end{array}$ & 0.506 & 0.170 \\
\hline$a_{1}$ & $\begin{array}{c}0.905 \\
(0.032) \\
\end{array}$ & 1.046 & 1.064 \\
\hline$a_{0}$ & $\begin{array}{c}1.746 \\
(0.031) \\
\end{array}$ & 3.337 & 1.760 \\
\hline$\tau_{e}$ & $\begin{array}{c}0.188 \\
(0.024) \\
\end{array}$ & 0.138 & 0.174 \\
\hline $1 / T_{\theta_{2}}$ & 0.30 & 1.67 & 0.40 \\
\hline$\zeta_{S P}$ & 0.34 & 0.29 & 0.40 \\
\hline$\omega_{S P}$ & 1.32 & 1.83 & 1.33 \\
\hline
\end{tabular}


Table 4 Tu-144LL LOES Modeling Summary Results - Phase Il Flight Tests

\begin{tabular}{|c|c|c|c|c|c|}
\hline & \multicolumn{3}{|c|}{ Longitudinal } & \multicolumn{2}{|c|}{ Lateral / Directional } \\
\hline & $\begin{array}{l}\text { Supersonic } \\
\text { Cruise }\end{array}$ & $\begin{array}{c}\text { Transonic } \\
\text { Cruise }\end{array}$ & $\begin{array}{l}\text { Canard } \\
\text { Extended } \\
\text { Approach }\end{array}$ & & $\begin{array}{l}\text { Supersonic } \\
\text { Cruise }\end{array}$ \\
\hline Maneuver(s) & $\mathrm{f} 23 \_1 \mathrm{a}$ & f2o_4b,c,d & 20_4h.i,j & Maneuver(s) & $\mathrm{f} 23,2 \mathrm{a}, 3 \mathrm{a}$ \\
\hline$M_{o}$ & 1.93 & 0.88 & 0.34 & $M_{\theta}$ & 1.94 \\
\hline$\alpha_{\theta}(\operatorname{deg})$ & 4.8 & 5.8 & 8.1 & $\alpha_{0}$ (deg) & 4.6 \\
\hline$h_{o}\left(10^{3} \mathrm{ft}\right)$ & 53.4 & 29.7 & 7.0 & $h_{\rho}\left(10^{3} \mathrm{ft}\right)$ & 53.9 \\
\hline$w(\mathrm{lbf})$ & $3.35 \times 10^{5}$ & $3.22 \times 10^{5}$ & $3.04 \times 10^{5}$ & $w(\mathrm{lhf})$ & $3.13 \times 10^{5}$ \\
\hline$I_{y}\left(\right.$ slug $\left.-\mathrm{ft}^{2}\right)$ & $1.44 \times 10^{7}$ & $1.37 \times 10^{7}$ & $1.28 \times 10^{7}$ & $I_{x}\left(\right.$ slug- $\left.\mathrm{ft}^{2}\right)$ & $1.17 \times 10^{6}$ \\
\hline$x_{i \cdot g}$ & $0.46 \bar{c}$ & $0.46 \bar{c}$ & $0.41 \bar{c}$ & $I_{z}\left(\operatorname{slug} g-\mathrm{ft}^{2}\right)$ & $1.51 \times 10^{7}$ \\
\hline Landing Gear & RET & RET & EXT & $l_{x z}\left(\right.$ slug-ft $\left.{ }^{2}\right)$ & $-9.75 \times 10^{4}$ \\
\hline Canard & RET & RET & EXT & $x_{c g}$ & $0.46 \bar{c}$ \\
\hline Nose (deg) & 0 & 0 & 17 & Landing Gear & RET \\
\hline$b_{1}$ & $0.353 \pm 0.011$ & $1.512 \pm 0.080$ & $0.626 \pm 0.026$ & Canard & RET \\
\hline$b_{0}$ & $0.106 \pm 0.006$ & $1.894 \pm 0.101$ & $0.457 \pm 0.154$ & Nose (deg) & 0 \\
\hline$a_{1}$ & $0.932 \pm 0.035$ & $3.680 \pm 0.182$ & $1.741 \pm 0.156$ & $c_{2}$ & $4.785 \pm 0.274$ \\
\hline$a_{0}$ & $1.970 \pm 0.035$ & $7.246 \pm 0.286$ & $1.518 \pm 0.380$ & $c_{1}$ & $3.464 \pm 0.116$ \\
\hline$\tau_{e}$ & $0.194 \pm 0.014$ & $0.280 \pm 0.009$ & $0.200 \pm 0.008$ & $c_{0}$ & $6.981 \pm 0.425$ \\
\hline$K_{\dot{\theta}}$ & 0.35 & 1.51 & 0.63 & $d_{2}$ & $-1.406 \pm 0.119$ \\
\hline $1 / T_{\theta_{2}}$ & 0.30 & 1.25 & 0.73 & $d_{1}$ & $0.490 \pm 0.184$ \\
\hline$\zeta_{S P}$ & 0.33 & 0.68 & 0.71 & $d_{0}$ & $2.377 \pm 0.158$ \\
\hline \multirow[t]{14}{*}{$\omega_{S P}$} & 1.40 & 2.69 & 1.23 & $e_{2}$ & $9.514 \pm 0.521$ \\
\hline & & & & $e_{1}$ & $1.052 \pm 0.113$ \\
\hline & & & & $e_{0}$ & $12.676 \pm 0.717$ \\
\hline & & & & $f_{2}$ & $0.278 \pm 0.060$ \\
\hline & & & & $f_{1}$ & $1.107 \pm 0.068$ \\
\hline & & & & $f_{0}$ & $0.263 \pm 0.033$ \\
\hline & & & & $g_{2}$ & $0.015 \pm 0.041$ \\
\hline & & & & $g_{1}$ & $-0.379 \pm 0.042$ \\
\hline & & & & $g_{0}$ & $1.082 \pm 0.084$ \\
\hline & & & & $\tau_{a}$ & $0.221 \pm 0.0 .39$ \\
\hline & & & & $\tau_{r}$ & $0.218 \pm 0.010$ \\
\hline & & & & $\zeta_{D R}$ & 0.169 \\
\hline & & & & $\omega_{D R}$ & 1.27 \\
\hline & & & & $1 / T_{R}$ & 4.34 \\
\hline $\begin{array}{c}\text { Mil-Std 1797A } \\
\text { FQ Level } \\
\end{array}$ & 2 & 3 & 2 & $\begin{array}{c}\text { Mil-Std 1797A } \\
\text { FQ Level } \\
\end{array}$ & 3 \\
\hline
\end{tabular}




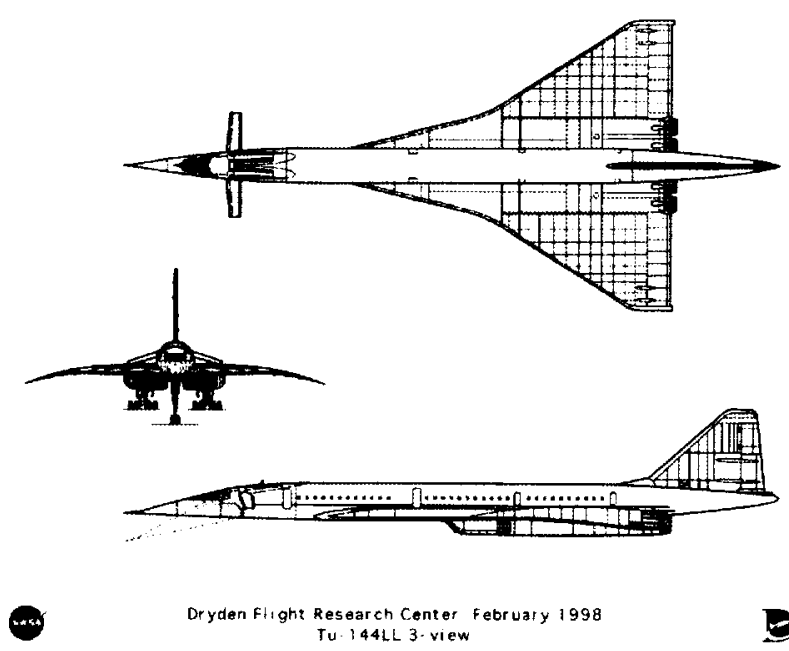

Figure 1 Tu-144LL 3-view
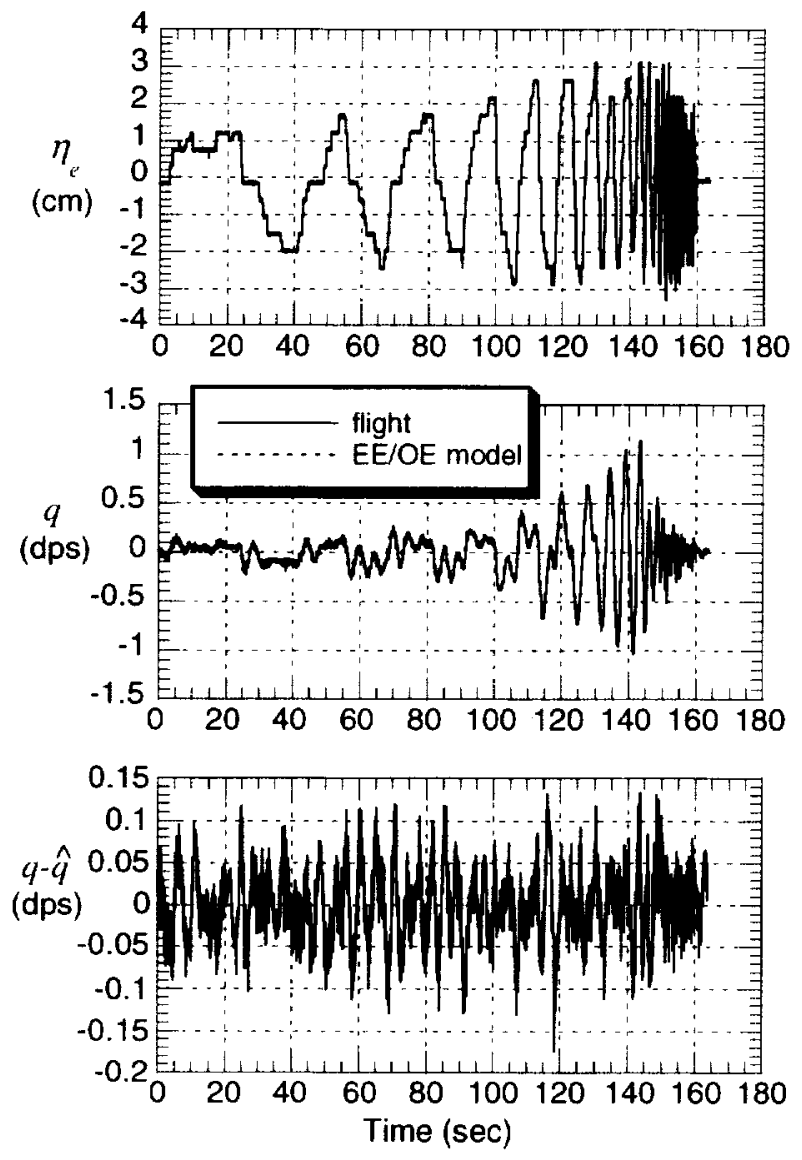

Figure 2 Frequency Sweep Maneuver 23_1a, $\alpha_{o}=4.8 \mathrm{deg} . M_{o}=1.93, x_{c g}=0.46 \bar{c}, I_{y}=1.44 \times 10^{7}$

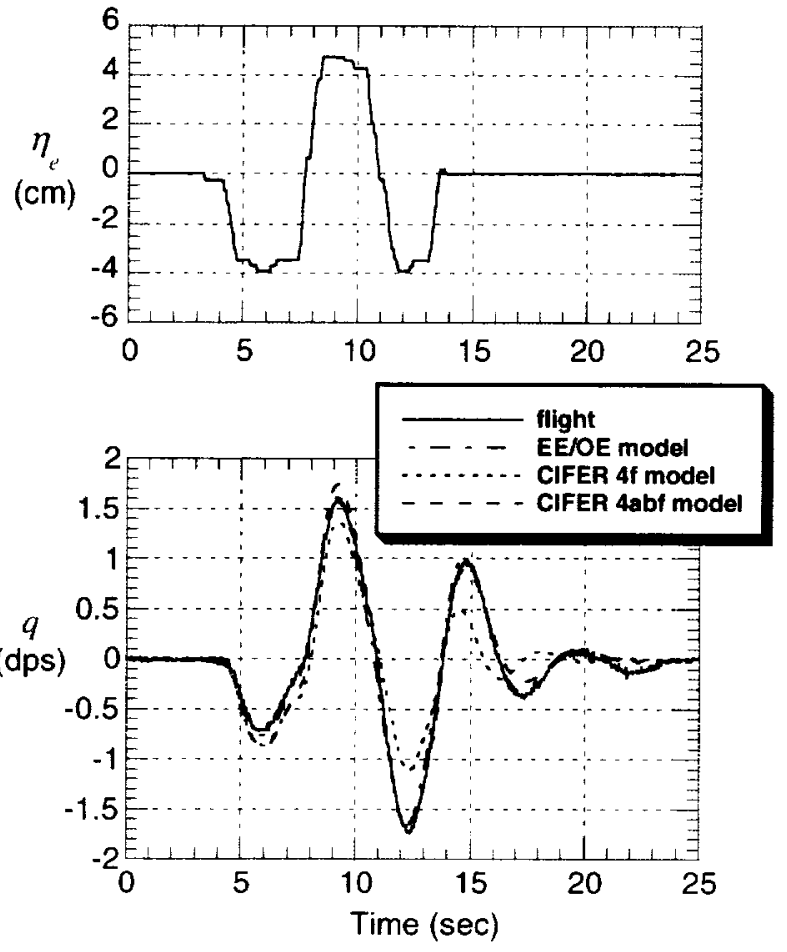

Figure 3 2-1-1 Maneuver 22_4f,

$\alpha_{o}=5.1 \mathrm{deg}, M_{O}=1.90, x_{c g}=0.46 \bar{c} \cdot l_{y}=1.41 \times 10^{7}$

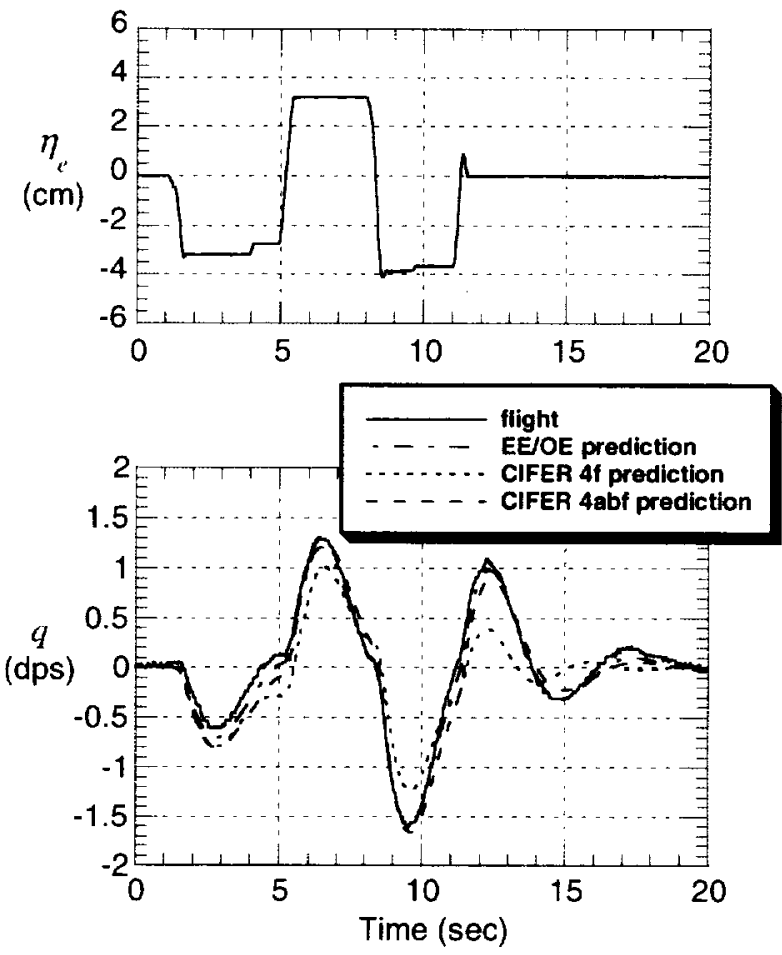

Figure 4 2-1-1 Prediction Maneuver 22_4a, $\alpha_{o}=5.1 \mathrm{deg}, M_{O}=1.91, x_{c g}=0.46 \bar{c}, I_{y}=1.44 \times 10^{7}$ 


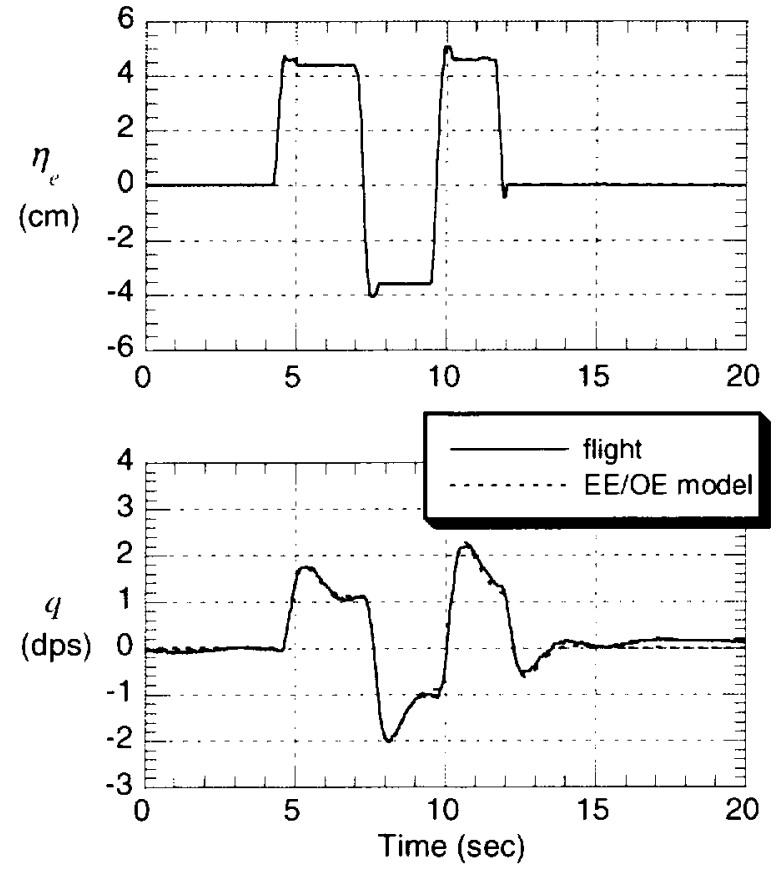

Figure 5 2-1-1 Identification Maneuver 20_4d, $\alpha_{o}=5.8 \mathrm{deg}, M_{o}=0.88, x_{c g}=0.46 \bar{c}, l_{y}=1.37 \times 10^{7}$
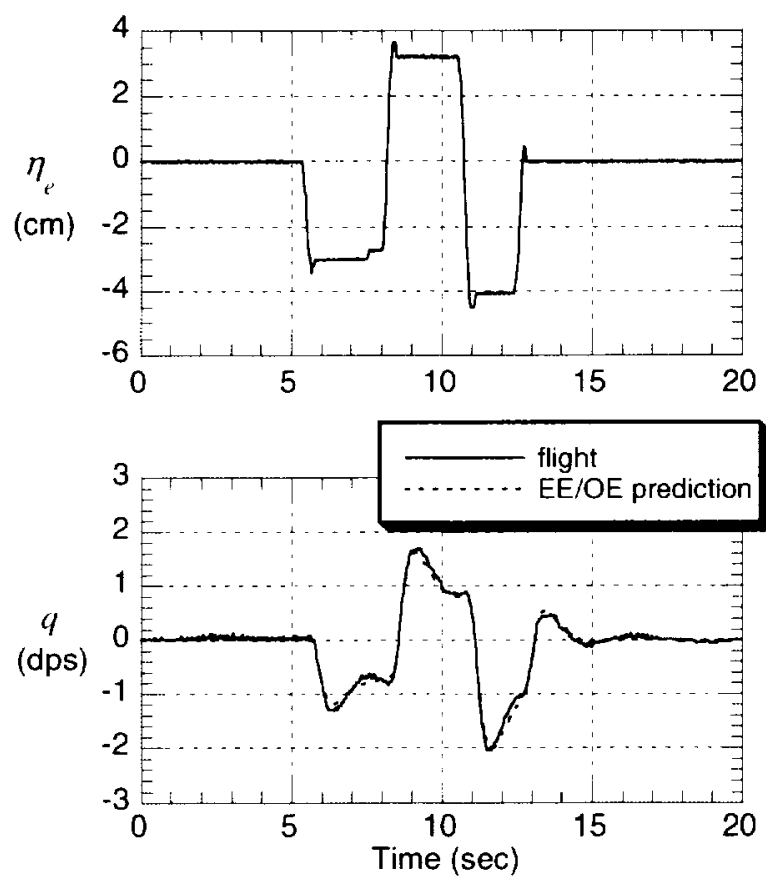

Figure 6 2-1-1 Prediction Maneuver 20_4f, $\alpha_{o}=5.8 \mathrm{deg}, M_{o}=0.88, x_{C g}=0.46 \bar{c}, I_{v}=1.37 \times 10^{7}$
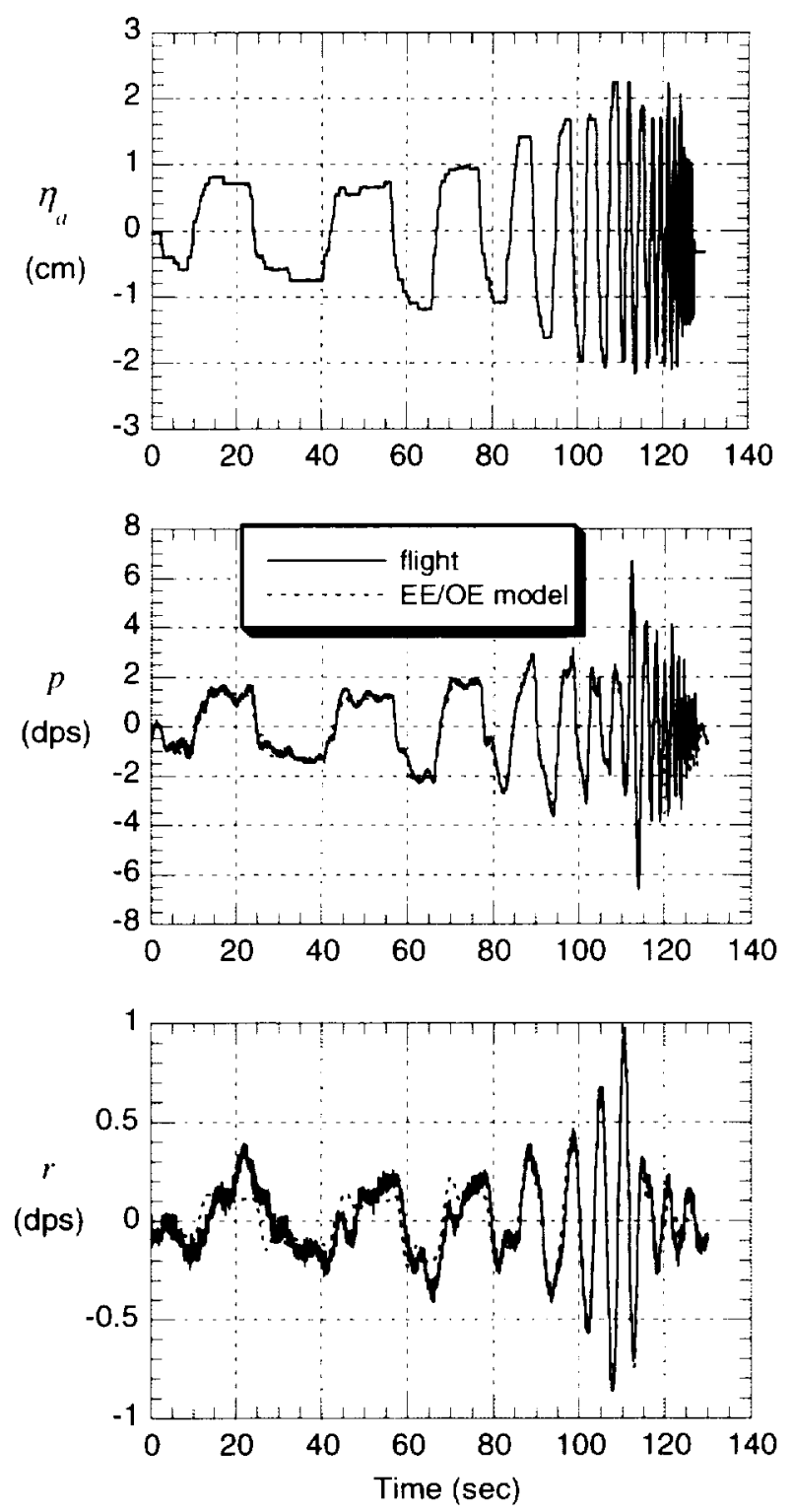

Figure 7 Frequency Sweep Maneuver 23_2a,

$$
\begin{gathered}
\alpha_{o}=4.6 \mathrm{deg}, M_{O}=1.94, x_{\mathrm{cg}}=0.46 \bar{c} . \\
I_{x}=1.17 \times 10^{6}, I_{z}=1.51 \times 10^{7}, I_{x z}=-9.75 \times 10^{4}
\end{gathered}
$$

